\title{
The Efficacy of Magnetic Resonance Imaging in The
}

\section{Determination Of Meniscus Tears in Patients With}

\section{Anterior Cruciate Ligament Tears}

\author{
Merve Gürsoy $^{1 *}$, Tuğrul Bulut ${ }^{2}$, Berna Dirim Mete ${ }^{1}$, Özgür Tosun $^{3}$, Emine Merve Horoz ${ }^{3}$, Safa Gürsoy ${ }^{4}$ \\ ${ }^{1}$ Department of Radiology, Izmir Democracy University Faculty of Medicine, Izmir, Turkey \\ ${ }^{2}$ Department of Orthopaedics and Traumatology, Izmir Katip Celebi University Ataturk Training and Research Hospital, Izmir, \\ Turkey \\ ${ }^{3}$ Department of Radiology, Izmir Katip Celebi University Ataturk. Training and Research Hospital, Izmir, Turkey \\ ${ }^{4}$ Department of Orthopaedics and Traumatology, Ankara Yildirim Beyazit University Faculty of Medicine, Ankara, Turkey
}

\begin{abstract}
The aim of this study was to determine the accuracy of MRI in meniscus and cruciate ligament (CL) pathologies. Another aim of the study was to determine the accuracy of MRI in the determination of meniscus tears in cases with and without ACL tear.

The study included 96 patients who were applied for meniscus and/or CL injury and examined by MRI between 2015-2018. The meniscus and CL were examined by MRI for findings of tears. The arthroscopy results were accepted as the gold standard and compared with the MRI results. The sensitivity, specificity, PPV, NPV, accuracy of MRI were calculated in the determination of meniscus and CL tears. The patients were also separated into 2 groups as those with ACL tear and without ACL tear. MRI accuracy in meniscus tear was compared between the 2 groups.

The sensitivity, specificity, PPV, NPV and accuracy rates of MRI in the evaluation of the medial meniscus tears were $93.5 \%, 88.8 \%$, $97.3 \%, 76.1 \%$ and $92.7 \%$ respectively. These values were $64.8 \%, 94.9 \%, 88.8 \%, 81.1 \%$ and $83.3 \%$ for the lateral meniscus (LM), $55.5 \%, 81.6 \%, 64.5 \%, 75.3 \%$ and $71.8 \%$ for ACL and $100 \%, 98.9 \%, 66.6 \%, 100 \%$ and $98.9 \%$ for posterior cruciate ligament. In the determination of LM tears, the specificity of MRI was significantly lower in the group with ACL tear ( $\mathrm{p}=0.021)$. No statistically significant difference was found in respect of the other values.

MRI has lower accuracy rates for ACL tear than for the meniscus. There was no significant difference in the accuracy of the MRI of meniscus tears between the groups with and without ACL tear.
\end{abstract}

Key Words: Magnetic Resonance Imaging, Arthroscopy, Meniscus, Anterior cruciate ligament, Accuracy

\section{Introduction}

Meniscus and the anterior cruciate ligament (ACL) tears are frequently seen pathologies. There are conflicting data in the literature about the accuracy of determining knee pathology in physical examination (1-3). Severe pain and swelling in patients with acute injuries may often prevent physical examination. This increases the importance of magnetic resonance imaging (MRI) in knee injuries. MRI is often the first method used in the diagnostic evaluation of meniscus and cruciate ligament injuries, thereby avoiding unnecessary diagnostic arthroscopy. Previous studies have reported MRI to have $79 \%-96 \%$ accuracy in the determination of meniscus tears and $92 \%-94 \%$ accuracy in cruciate ligament tears (4-7).

There are various factors that affect false positive and false negative diagnoses in MRI. Tears located in the meniscus posterior horn have been reported to be difficult to diagnose arthroscopically. Therefore, some of the false positive results may be cases that have been missed in arthroscopy (7). Previous studies in the literature have reported that the determination of meniscus tears concomitant to ACL injuries is even more difficult (8). In a study by Nam et al. (9) the sensitivity and negative predictive value (NPV) of MRI were determined to be lower in patients with ACL tear compared to those without.

The aim of this study was to determine the sensitivity, specificity, positive predictive value (PPV), negative predictive value (NPV) and accuracy rates of MRI in the meniscus and cruciate ligament pathologies by comparing these values with arthroscopic findings. A secondary aim of the study was to determine the accuracy of MRI in the determination of meniscus tears in cases with and without ACL tears. 
Table 1. Accuracy of MRI in the diagnosis of meniscal and ligamentous tears (the data is given as the percentage)

\begin{tabular}{lccccc}
\hline & Sensitivity & Specificity & $\begin{array}{c}\text { Positive predictive } \\
\text { value }\end{array}$ & $\begin{array}{c}\text { Negative } \\
\text { predictive value }\end{array}$ & Accuracy \\
\hline Medial Meniscus & 93.5 & 88.8 & 97.3 & 76.1 & 92.7 \\
Lateral Meniscus & 64.8 & 94.9 & 88.8 & 81.1 & 83.3 \\
Anterior cruciate & 55.5 & 81.6 & 64.5 & 75.3 & 71.8 \\
ligament & 100 & 98.9 & 66.6 & 100 & 98.9 \\
$\begin{array}{l}\text { Posterior cruciate } \\
\text { ligament }\end{array}$ & & & & & \\
\hline
\end{tabular}

Table 2. MRI results obtained by taking arthroscopy results as the gold standard

\begin{tabular}{lcccc}
\hline & $\begin{array}{c}\text { MM tear without } \\
\text { ACL tear }\end{array}$ & $\begin{array}{c}\text { MM tear with } \\
\text { ACL tear }\end{array}$ & $\begin{array}{c}\text { LM tear without } \\
\text { ACL tear }\end{array}$ & $\begin{array}{c}\text { LM tear with } \\
\text { ACL tear }\end{array}$ \\
\hline True positive & 48 & 25 & 16 & 8 \\
True negative & 8 & 8 & 34 & 22 \\
False positive & 2 & 0 & 1 & 2 \\
False negative & 2 & 3 & 9 & 4 \\
\hline
\end{tabular}

MM: Medial meniscus, LM: Lateral meniscus, ACL: Anterior cruciate ligament

Table 3. Diagnostic accuracies of MRI in the determination of medial and lateral meniscus tears

\begin{tabular}{lccccc}
\hline & Sensitivity (\%) & Specificity (\%) & PPV (\%) & NPV (\%) & Accuracy (\%) \\
MM tear without ACL tear & 96.0 & 80.0 & 96.0 & 80.0 & 93.3 \\
MM tear with ACL tear & 89.2 & 100 & 100 & 72.7 & 91.6 \\
P value & 0.250 & 1.000 & 0.090 & 0.360 & 0.453 \\
LM tear without ACL tear & 64.0 & 97.1 & 94.1 & 79 & 83.3 \\
LM tear with ACL tear & 66.6 & 91.6 & 80 & 84.6 & 83.3 \\
P value & 0.687 & 0.021 & 0.098 & 0.650 & 0.210 \\
\hline
\end{tabular}

MM: Medial meniscus, LM: Lateral meniscus, ACL: Anterior cruciate ligament,

PPV: Positive predictive value, NPV: Negative predictive value

\section{Materials and Methods}

This retrospective study was approved by the Institutional Review Board of our hospital (IRB number: 314). Since the nature of the study was retrospective, informed consent by patients was not required.

The study included patients who underwent arthroscopy due to a meniscus and/or cruciate ligament injury between January 2015 and January 2018 in the Orthopaedics and Traumatology Clinic of our hospital. Patients were excluded if they had previously undergone open knee surgery or arthroscopy for any reason, had severe degenerative changes, had undergone MRI examination at another center or were aged $<18$ years. With the application of these exclusion criteria, 56 of 152 patients were excluded from the study. A total of 96 patients were included in the study for evaluation.
The knee MRI examination was made with a 1.5 Tesla MRI device (General Electric Medical Systems, Milwaukee, WI, USA) using a knee coil. For all the cases, MRI was applied using standard sagittal proton density fat-suppressed turbo spin echo (TSE) sequences (TR/TE: 1,500/15, matrix: 256x384, $18 \mathrm{~cm}$ FOV), coronal proton density fat-suppressed TSE (TR/TE: 1,500/15, matrix: 256x320, $18 \mathrm{~cm}$ FOV), coronal T1-weighted TSE (TR/TE: 500/18, matrix: 256x320, $21 \mathrm{~cm}$ FOV) and axial T2-weighted fatsuppressed TSE (TR/TE: 2000/60, matrix: 256x288, $16 \mathrm{~cm}$ FOV). Slice thickness was $3 \mathrm{~mm}$. The knee MR images were evaluated retrospectively by a radiologist specialized in musculoskeletal system radiology. The radiologist was not aware of arthroscopy findings. The Stoller et al. (10) classification system was used in the evaluation of the meniscus on MRI. Cases with Grades 1 and 2 on MRI were accepted as the group with no meniscus tear, and cases with Grade 3 as the group with a meniscus tear. 


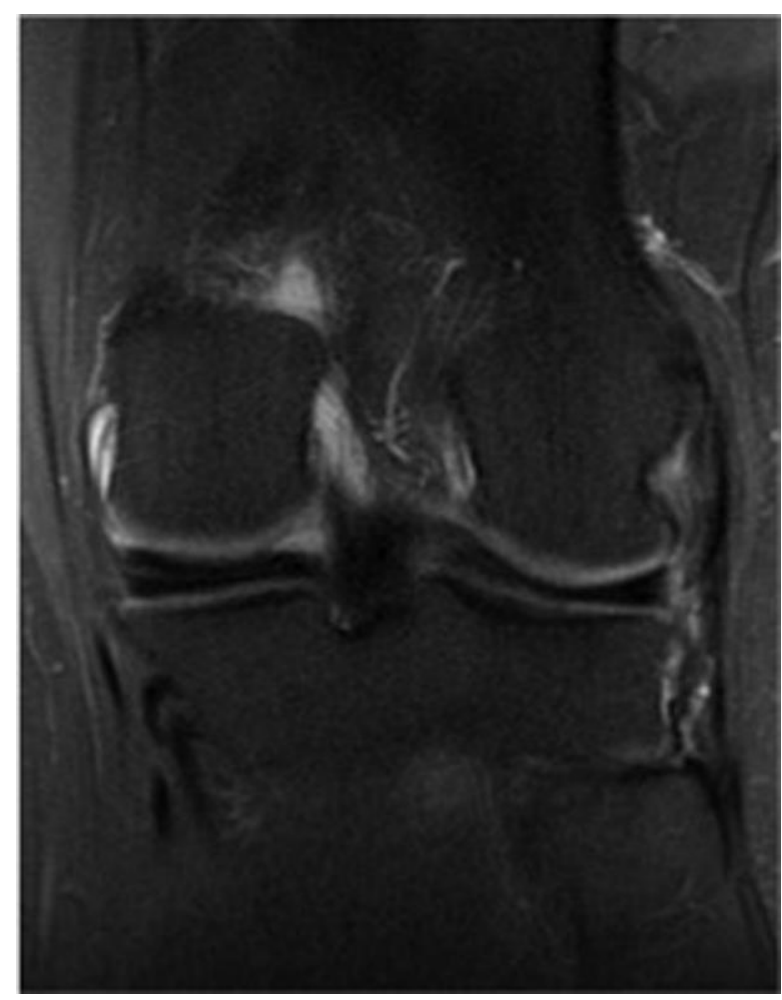

a

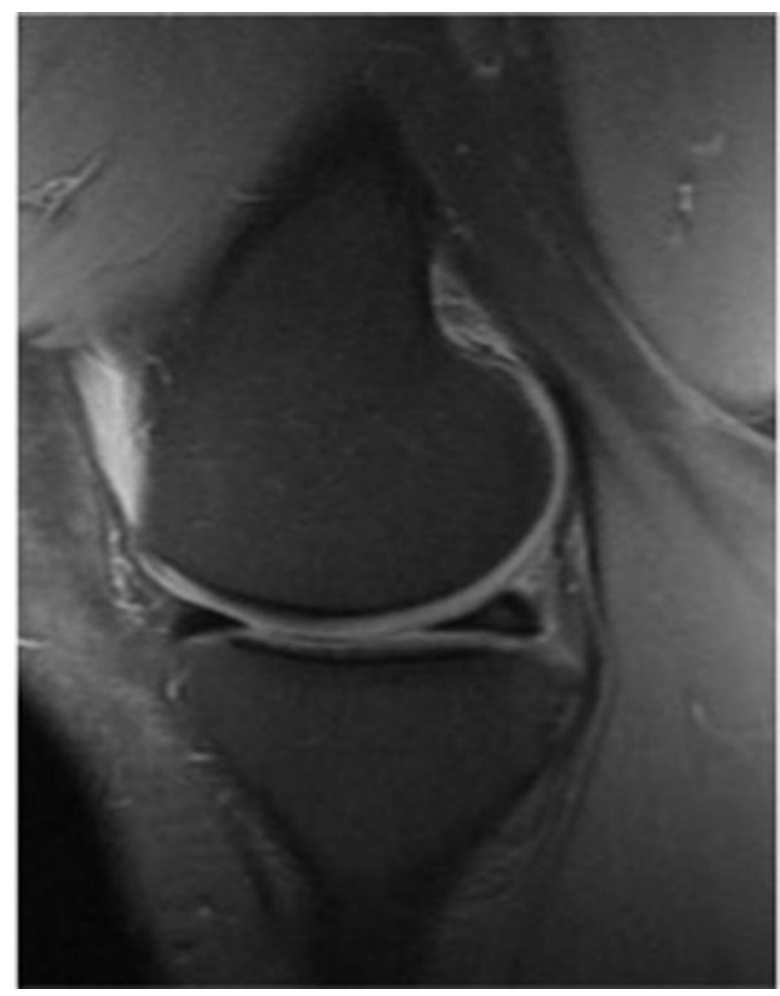

b

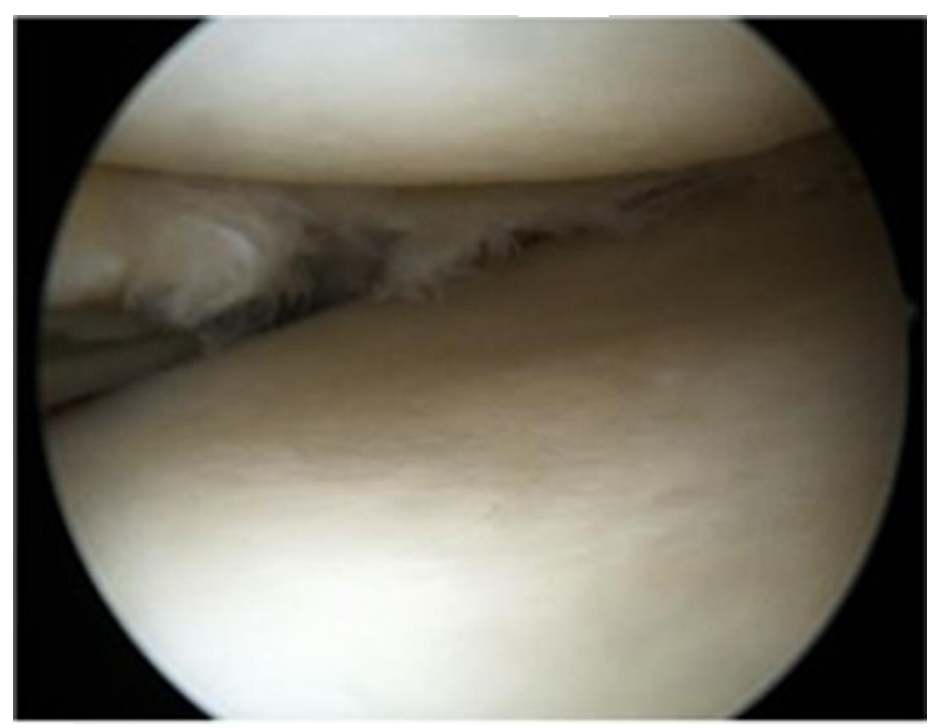

c

Fig. 1. Coronal proton density fat-suppressed TSE (a) and sagittal proton density fat-suppressed TSE (b) images showed grade 2 signal change in the medial meniscus posterior horn. Arthroscopy (c) revealed a complex tear in the same location, which was invisible on MRI. It was accepted as a false negative result of MRI

In the evaluation of cruciate ligaments, cases observed with a normal cruciate ligament or a degenerative signal increase without a tear on MRI were included in the group without a tear and those with a partial or complete tear formed the group with a tear. (11) Pathologies determined from retrospective examination of the arthroscopy reports were recorded. The orthopedist who applied the arthroscopy was aware of the MRI findings. In all cases, there was less than 8 weeks between MRI and arthroscopy.

The arthroscopy results were accepted as the gold standard and were compared with the MRI results. The sensitivity, specificity, PPV, NPV and accuracy rates of MRI were calculated in the determination of meniscus and cruciate ligament tears. The patients were separated into two groups as those with ACL tear and those without ACL tear. The analysis was made of whether or not there was any difference in 
the reliability of MRI between the two groups in the determination of meniscus tears.

Statistical Analysis: All the data were analyzed using SPSS version 20.0 software. The sensitivity, specificity, PPV, NPV and accuracy of MRI for medial meniscus (MM) and (LM) tears were compared and analyzed statistically. Sensitivity was calculated as the number of true positive examinations, divided by the number of true positive plus false negative examinations. Specificity was calculated as the number of true negative examinations, divided by the number of true negative plus false positive examinations. PPV was calculated as the number of true positive examinations, divided by the number of all true and false positive examinations. NPV was calculated as the number of true negative examinations, divided by the number of all true and false negative examinations. Accuracy was calculated as the number of true positive plus true negative examinations, divided by the number of all cases.

McNemar chi-square test was used to compare the diagnostic accuracies. A value of $\mathrm{p}<0.05$ was accepted as statistically significant.

\section{Results}

The total of 96 cases included in the study comprised 62 males and 34 females with a mean age of 41.9 years (range, 19-65 years).

In the evaluation of the MRI results, there were determined to be 2 false positives, 5 false negatives, 73 true positives and 16 true negatives in the medial meniscus (MM) (Figure 1). In the lateral meniscus $(\mathrm{LM})$, there were 3 false positives, 13 false negatives, 24 true positives, and 56 true negatives. In the ACL, there were 11 false positives, 16 false negatives, 20 true positives, and 49 false negatives. In the posterior cruciate ligament (PCL), there was 1 false positive, 0 false negatives, 2 true positives, and 93 true negatives. The sensitivity, specificity, PPV, NPV and accuracy rates of MRI in the evaluation of the meniscus and cruciate ligament pathologies are shown in (Table 1).

The 96 patients included in the study were separated into two groups as those with ACL tear and those without. ACL tear was determined in 36 patients and not in 60 patients. MM tears were determined in $86.6 \%$, and LM tears in $41.6 \%$ of the group without ACL tear. In the group with ACL tear, MM tear was determined in $80.5 \%$ and LM tear in $33.3 \%$ of the patients.

True positive, true negative, false positive and false negative results of MRI were obtained by taking arthroscopy results as the gold standard (Table 2).
The sensitivity, specificity, PPV, NPV and accuracy rates of MRI in the determination of MM and LM tears in the groups with and without ACL tears are shown in (Table 3). No significant difference was determined between the groups in the determination of MM in respect of the sensitivity, specificity, PPV, NPV and accuracy rates of MRI. In the determination of LM tears, the specificity of MRI was determined to be statistically significantly lower in the group with ACL tear $(p=0.021)$. There was no statistically significant difference in respect of the other values.

\section{Discussion}

MRI currently has a place in diagnostic arthroscopy and has become the first method to be requested in the determination of knee pathologies. In our study, the sensitivity and PPV of MRI in the determination of $\mathrm{MM}$ tears were $>90 \%$ and specificity was $88 \%$, which were similar rates to those previously reported in the literature. NPV was $76.1 \%$, which was lower than the rates in the literature (12). This low NPV in the determination of MM tears was caused by 5 cases with a false negative result on MRI. In 3 of these cases, the tear was in the posterior horn and in 2 cases, in the anterior horn. In 1 case with degeneration and evident thinning of the $\mathrm{MM}$ at the level of the posterior horn and the ligament root junction on MRI, there was a radial tear reported on arthroscopy. The junction of the posterior horn and ligament root is one of the frequent localization areas of a radial tear. As this junction is thin, it may not have entered the slice thickness and the radial tear may not have been visualized (13). In 1 case with grade 2 degeneration in the MM posterior horn on MRI, a complex tear was determined on arthroscopy. In another case with an intrasubstance degenerative signal in the MM posterior horn observed on MRI, a short segment complex tear was seen on arthroscopy. Grade 1 degeneration in the MM anterior horn was observed on MRI in 2 cases, 1 of whom had a radial tear and the other a complex tear on arthroscopy. The time that elapsed between MRI and arthroscopy could have been a reason that these tears were not seen on MRI.

When the LM was examined, the sensitivity of MRI was $64.8 \%$, which was low compared to previous reports in the literature (4). Specificity, PPV and NPV were consistent with literature $(94.9 \%, 88.8 \%, 81.1 \%$, respectively). Similar to the current study, Oei et al. (14) reported that MRI was more sensitive in the determination of MM tears and more specific for LM tears. When the false positive results were examined in the current study, there were 2 for MM and 3 for 
LM. Of the total 5 false positive results, $4(80 \%)$ were in the posterior horn. In a study by Mink et al. (15) $70 \%$ of the false positive cases were reported to be tears in posterior horn localization. Munk et al. (16) reported that all false positive cases of an examined series were localized in the posterior horn. In arthroscopy, difficulties may be experienced in the visualization of the posterior horn (1). Therefore, the false negativity in arthroscopy could be reflected in MRI studies in the form of false positivity. Normal anatomic structures such as inferior geniculate artery, popliteal hiatus, subpopliteal recess and, Humphrey ligament may be incorrectly evaluated as LM tear (17).

In our study, the highest rates of false positive and false negative results were determined in ACL tears. The accuracy of the determination of ACL tears on MRI in the current study was found to be low compared to previous reports in the literature, which have stated this rate to be $>90 \% .[18,19]$ In the current study, the routine MRI tests were examined retrospectively. In our routine knee MRI tests, it is aimed for the time of the patient remaining completely still inside the MRI unit to be kept to the optimum duration. Therefore, SE T2-weighted sagittal slices were not taken in addition to the PD sequence with coronal, oblique and axial oblique slices set parallel to the ACL course. Adding this plane and sequences to the MRI plan significantly increases the diagnostic accuracy, especially in cruciate ligament pathologies. (20) These sequences are added to the examination plan in the clinical request for patients with a known initial diagnosis of ACL tear. However, these patients were not included in the study group. In addition, uncertainties may be experienced in the differentiation of partial and complete ACL tears on MRI (21), which can be one of the factors affecting the low accuracy rate. That the radiologist was blinded to the clinical findings of the study could be considered to have affected the low rate.

There are several studies in literature related to meniscus tears accompanying ACL damage. (22) Nam et al. (9) reported that ACL tears were accompanied by MM tears at the rate of $59 \%$ and by LM tears at $44 \%$. In our study, $80.5 \%$ of ACL tears were accompanied by MM tears and $33.3 \%$ by LM tears, respectively. For MM, the rate was extremely high in comparison with literature, but for LM the rate was lower. It is thought that the use of different definitions for meniscus tears in arthroscopy could affect these differences in rates.

The accuracy of MRI in PCL pathologies was extremely high in the current study at $98.9 \%$. Although the number of patients with PCL tear was low, this result was consistent with the literature. (23,

\section{4)}

In the current study, it was aimed to compare the accuracy of MRI in the determination of meniscus tears in groups with and without ACL tears. It was predicted that the accuracy of MRI in predicting meniscus tears could be lower in cases with a concomitant ACL tear. The specificity of MRI in determining LM tears was statistically significantly lower in the group with ACL tear. However, no difference was observed when looking at other parameters. De Smet et al. (8) reported that when there was ACL damage, the sensitivity of MRI in the determination of MM tears fell from $97 \%$ to $88 \%$, and the sensitivity in the determination of LM tears fell from $94 \%$ to $69 \%$. Jee et al. (25) stated that sensitivity was lower for MM and LM, Sampson et al. (26) stated that sensitivity was lower for LM, in cases with concomitant ACL damage.

In a study by Lundberg et al. (27) it was reported that the accuracy of MRI was reduced in knee pathologies seen together with hemarthrosis, and this decrease could be related to the catabolic processes during hemoglobin destruction in the meniscus and chondral tissues.

In the current study, there was no significant difference between the groups with and without ACL tear in respect of the accuracy of the determination of meniscus tears. Specificity in the determination of LM tears was significantly lower in the group with ACL tear. A reason for this could have been the formation of ACL tears by anteromedial and anterolateral rotator instability type injuries. At the same time, damage can often develop in the posterolateral corner structures of the knee. The low rate of damage of the popliteal tendon, popliteomeniscal fascicles, the fibular collateral ligament and other posterolateral corner ligaments in close proximity to the LM can be considered to have had an effect. Soft tissue edema and blood products at these levels and contusional signal changes accompanying LM can be considered to contribute to the reduction in rate.

That the radiologist was unaware of the clinical findings when evaluating the MRI is a limitation of the study and could have caused the lower rates compared to literature. Another limitation of the study was its retrospective nature. As there were few cases with PCL tear, there is a need for further studies with a greater number of patients to provide statistical results on this subject. The strongest aspect of this study was that the reliability of MRI was evaluated by comparing groups with and without ACL tear, as the majority of previous studies have been conducted without a control group. $(25,26,28)$

In conclusion, MRI is a non-invasive method that can 
be safely used in the diagnosis of lateral and medial meniscus tears. MRI has better diagnostic accuracy for meniscal tears than ACL tears. This study showed that if a patient had an ACL tear, the specificity of MRI for LM tear were less than if there was no ACL tear. However, there was no significant difference in the accuracy rates of the MRI evaluation of meniscus tears between the group with ACL tear and those without ACL tear.

\section{References}

1. Rose NE, Gold SM. A comparison of accuracy between clinical examination and magnetic resonance imaging in the diagnosis of meniscal and anterior cruciate ligament tears. Arthroscopy 1996; 12: 398-405.

2. Thomas S, Pullagura M, Robinson E, Cohen A, Banaszkiewicz P. The value of magnetic resonance imaging in our current management of ACL and meniscal injuries. Knee Surg Sports Traumatol Arthrosc 2007; 15: 533-536.

3. Navali AM, Bazavar M, Mohseni MA, Safari B, Tabrizi A. Arthroscopic Evaluation of the Accuracy of Clinical Examination versus MRI in Diagnosing Meniscus Tears and Cruciate Ligament Ruptures. Arch Iran Med 2013; 16: 229232.

4. Behairy NH, Dorgham MA, Khaled SA. Accuracy of routine magnetic resonance imaging in meniscal and ligamentous injuries of the knee: Comparison with arthroscopy. Int Orthop 2009; 33: 961-967.

5. Fox MG. MR imaging of the meniscus: Review, current trends, and clinical implications. Radiol Clin North Am 2007; 45: 1033-1053.

6. Crawford R, Walley G, Bridgman S, Maffulli N. Magnetic resonance imaging versus arthroscopy in the diagnosis of knee pathology, concentrating on meniscal lesions and ACL tears: A systematic review. Br Med Bull 2007; 84: 5-23.

7. Bin Abd Razak HR, Sayampanathan AA, Koh TH, Tan HC. Diagnosis of ligamentous and meniscal pathologie $s$ in patients with anterior cruciate ligament injury: comparison of magnetic resonance imaging and arthroscopic findings. Ann Transl Med 2015; 3: 243.

8. De Smet AA, Graf BK. Meniscal tears missed on MR imaging: Relationship to meniscal tear patterns and anterior cruciate ligament tears. AJR Am J Roentgenol 1994; 162: 905-911.

9. Nam TS, Kim MK, Ahn JH. Efficacy of magnetic resonance

imaging evaluation for meniscal tear in acute anter ior cruciate ligament injuries. Arthroscopy 2014; 30: 475-482.

10. Stoller DW, Martin C, Crues JV, Kaplan L, Mink
JH. Meniscal tears: Pathologic correlation with MR imaging. Radiology 1987; 163: 731-738.

11. Stoller DW, Cannon WD, Lesley JR. Magnetic resonance imaging in orthopedics and sport medicine. In: Stoller D (edi). The knee. Philadelphia: Lipponcott Williams 1997; 204-205.

12. Sanches Vas CE, de Camargo O, de Santana P Valezi AC. Accuracy of magnetic resonance in identifying traumatic intraarticularknee lesions. Clinics (Sao Paulo) 2005; 60: 445-450.

13. LaPrade RF, Ho CP, James E, Crespo B, LaPrade CM, Matheny LM. Diagnostic accuracy of $3.0 \mathrm{~T}$ magnetic resonance imaging for the detection of meniscus posterior root pathology. Knee Surg Sports Traumatol Arthrosc 2015; 23: 152-157.

14. Oei E, Nikken J, Verstijnen AC, Ginai AZ, Myriam Hunink MG. MR imaging of the menisci and cruciate ligaments: a systematic review. Radiology 2003; 226: 837-848.

15. Mink JH, Levy T, Crues III JV. Tears of the anterior cruciate ligament and menisci of the knee: MR imaging evaluation. Radiology 1988; 167: 769-774.

16. Munk B, Madsen F, Lundorf E, et al. Clinical magnetic resonance imaging and arthroscopic findings in knees: a comparative study of meniscus anterior prospective cruciate ligament and cartilage lesions. Arthroscopy 1998; 14: 171-175.

17. Herman LJ, Beltran J. Pitfalls in MR imaging of the knee. Radiology 1988; 167: 775-781.

18. Jackson DW, Jennings LD, Maywood RM, Berger PE. Magnetic resonance imaging of the knee. Am J Sports Med 1988; 16: 29-38.

19. Ozturan KE, Yucel I, Cakıc1 H, Guven M, Gurel K. Menisküs Ve Bağ Yaralanmalarının Manyetik Rezonans Görüntüleme Ve Artroskopik Cerrahi Bulgularının Karşılaştırılması. Düzce Tıp Derg 2012; 14: 1-4.

20. Ng AW, Griffith JF, Hung EH, Law KY, Yung PS. MRI diagnosis of ACL bundle tears: value of oblique axial imaging. Skeletal Radiol 2013; 42: 209-217.

21. Cavinatto L, Gupta S, Morgan C, Bartolozzi AR. Value of Preoperative MRI and Examination under Anesthesia for Differentiating Complete from Partial Anterior Cruciate Ligament Tears. J Knee Surg 2018 Jul 10.

22. Dufka FL, Lansdown DA, Zhang AL, Allen $\mathrm{CR}, \mathrm{Ma} \mathrm{CB}$, Feeley BT. Accuracy of MRI evaluation of meniscus tears in the setting of ACL injuries. Knee 2016; 23: 460464.

23. Gross ML, Grover JS, Bassett LW, Seeger LL, Finerman GA. Magnetic resonance imaging of the posterior cruciate ligament. Clinical use to improve diagnostic accuracy. Am J Sports Med 1992; 20: 732-737. 
24. Trieshmann HW Jr., Mosure JC. The impact of magnetic resonance imaging of the knee on surgical decision making. Arthroscopy 1996; 12: $550-555$

25. Jee WH, McCauley TR, Kim JM. Magnetic resonance diagnosis of meniscal tears in patients with acute anterior cruciate ligament tears. J Comput Assist Tomogr 2004; 28: 402-406.

26. Sampson MJ, Jackson MP, Moran CJ, Shine S, Moran R, Eustace SJ. Three Tesla MRI for the diagnosis of meniscal and anterior cruciate ligament pathology: A comparison to arthroscopic findings. Clin Radiol 2008; 63: 1106-1111.
27. Lundberg $M$, Odensten $M$, Thuomas KA, Messner K. The diagnostic validity of magnetic resonance imaging in acute knee injuries with hemarthrosis. A single-blinded evaluation in 69 patients using high-field MRI before arthroscopy. Int J Sports Med 1996; 17: 218-222.

28. Laundre BJ, Collins MS, Bond JR, Dahm DL, Stuart MJ, Mandrekar JN. MRI accuracy for tears of the posterior horn of the lateral meniscus in patients with acute anterior cruciate ligament injury and the clinical relevance of missed tears. AJR Am J Roentgenol 2009; 193: 515-523. 